Original Article

\title{
The effects of neuromuscular joint facilitation treatment using neck patterns on the acoustic analysis of swallowing sounds in healthy adults
}

\author{
Xiao Wen, MSc ${ }^{1,2)}$, Luping Li, MSc ${ }^{3)}$, Ko OnOdA, RPT, $\mathrm{PhD}^{3)}$, \\ Hitoshi Maruyama, RPT, $\mathrm{PhD}^{4}$ ) \\ 1) Narita Campus, International University of Health and Welfare, Japan \\ 2) Department of Speech, Language and Hearing Sciences, China Rehabilitation Research Center: \\ No. 10, Jiaomen North Road, Fengtai District, Beijing 100068, China \\ 3) Department of Physical Therapy, School of Health Sciences, International University of Health and \\ Welfare, Japan \\ 4) Fukuoka Campus, International University of Health and Welfare, Japan
}

\begin{abstract}
Purpose] We aimed to investigate the changes in the swallowing sounds of healthy adults during neuromuscular joint facilitation treatment using neck patterns. [Participants and Methods] A total of 20 healthy adults (10 males and 10 females; mean age, $29.2 \pm 6.3$ years) swallowed $10 \mathrm{~mL}$ of water four times under three conditions (after the neuromuscular joint facilitation neck-flexion resistance pattern, after the Shaker-type exercise, and during relaxed sitting without prior exercise [control]), randomly ordered with an interval greater than 3 days. Swallowing sounds for each water swallow were recorded using cervical auscultation. [Results] The mean amplitude of swallowing sound intensity and the mean spectral frequency were significantly higher after the neuromuscular joint facilitation neck-flexion resistance pattern and the Shaker-type exercise, in comparison with those in the control group. [Conclusion] Neuromuscular joint facilitation training with the neck-flexion resistance pattern influenced swallowing sounds to the same degree as the Shaker-type exercise, implying that this resistance pattern may enhance suprahyoid muscle contraction.

Key words: Neuromuscular joint facilitation, Shaker exercise, Swallowing sound
\end{abstract}

(This article was submitted Jun. 14, 2021, and was accepted Jul. 26, 2021)

\section{INTRODUCTION}

The Shaker exercise, a series of sustained and repetitive head lifting with isometric and isotonic tasks, is frequently used in rehabilitation for swallowing disorders to enhance suprahyoid and infrahyoid muscle strength ${ }^{1,2)}$, resulting in improved superior-anterior movement of the hyoid and a wider upper esophageal sphincter (UES) opening ${ }^{2-4)}$. Furthermore, strengthening the pharyngeal muscle activity can decrease the amount of residue in the piriform sinus and prevent aspiration ${ }^{5,6)}$. In the Shaker exercise, the isometric tasks were performed by three repetitions of raising the head for 1 minute followed by a 1-min rest, and the isotonic tasks were performed by 30 repetitions of alternate up-and-down movement of the head.

Despite the above-mentioned benefits, the Shaker exercise has been considered physically challenging, especially for the elderly and those with motor dysfunction ${ }^{7,8)}$, affecting compliance. Therefore, modifications, such as the modified Shaker exercise by Babu et al., chin tuck posture against resistance (CTAR) by Yoon et al., and recline and head-lift exercises by Fujiki et al. have been proposed ${ }^{6,9,10)}$. These Shaker-type neck flexion exercises are known to effectively strengthen the suprahyoid and infrahyoid muscles.

*Corresponding author. Xiao Wen (E-mail: 18S1041@g.iuhw.ac.jp)

(C2021 The Society of Physical Therapy Science. Published by IPEC Inc.

(c) (1) $\odot$ This is an open-access article distributed under the terms of the Creative Commons Attribution Non-Commercial No DerivaCC BY NC ND tives (by-nc-nd) License. (CC-BY-NC-ND 4.0: https://creativecommons.org/licenses/by-nc-nd/4.0/) 
Neuromuscular joint facilitation (NJF) is a new therapeutic exercise based on the kinesiology that integrates proprioceptive neuromuscular facilitation (PNF) with joint composition movement, through passive, active, and resistance exercises. It aims to improve joint movement and increase strength, flexibility, and range of motion (ROM). NJF is a dynamic approach for simultaneously evaluating and treating the nerve, muscle, and joint. The NJF neck pattern treatment, which includes neck forward flexion, lateral flexion, and head rotation resistance training, is used to improve the neck muscle proprioception, increasing muscle force and active $\mathrm{ROM}^{11)}$.

To our knowledge, there has been no study showing the effects of NJF on swallowing function. The NJF technique has similar or better effects on swallowing, as compared with Shaker-type exercises was hypothesized. We aimed to compare the effects of a Shaker-type exercise and NJF neck flexion resistance pattern (NJF-NFRP) on swallowing sounds in healthy adults.

\section{PARTICIPANTS AND METHODS}

A total of 20 healthy adults (10 males and 10 females) aged 22 to 35 years (mean, $29.2 \pm 6.3$ years) were recruited from the International University of Health and Welfare. The detailed characteristics of the participants are shown in Table 1.

The study objectives and contents were explained to all participants prior to recruitment, and informed consent was obtained. All experimental procedures in this study were reviewed and approved by the Ethical Review Committee of the International University of Health and Welfare (IRB No. 18-Io-108). All the procedures performed were in accordance with the ethical standards of the institution.

Participants were randomly assigned to groups with one of three different experimental conditions (NJF, Shaker-type exercise, and during relaxed sitting without prior exercise [control]). After intervals of $>3$ days, they were re-assigned randomly, until each participant was tested under all three different conditions.

The NJF neck pattern treatment is commonly applied five times continuously on each side ${ }^{11)}$. The interventions were performed by the same qualified physical therapist to avoid individual variations in treatment. Five repetitions were performed on each side of the neck using the NFRP, one of two NJF neck pattern treatments (namely, flexion and extension) (Fig. 1).

To ensure consistency in duration and intensity between the Shaker-type and NJF interventions, the intensity of the traditional Shaker exercise was modified. Participants in the supine position were instructed to raise their head high enough to observe their toes, without raising their shoulders, for 5 seconds, instead of 1 min as generally done, followed by a 5-s rest. The task was repeated for nine times.

This study design considers the sitting position as the most common eating position for humans. During the whole measurement process, all three groups adopted comfortable sitting positions while swallowing. Before recording the swallowing sound, the assistant injected $10 \mathrm{~mL}$ of distilled water into the floor of the participants' mouths using a syringe, and asked them to hold it until the command to swallow was given.

Before the interventions, the auscultation positions were marked on the left and right sides of the neck, above the cricoid cartilage, in front of the sternocleidomastoid muscle and the large vessels ${ }^{12-14)}$. The examiner gently placed the electronic stethoscope (Littmann Model 3200; 3M, MN, USA) on the marked positions on the neck to collect the swallowing sounds. In the NJF-NFRP condition, the swallowing sound was recorded during resistance in the isometric contraction of the middle limb. In the Shaker-type exercise condition, the swallowing sound was recorded during isometric neck flexion ${ }^{6}$. In the control condition, the swallowing sound was recorded during relaxed sitting in a chair.

The swallowing sounds were recorded four times (twice on each side of the neck) for each condition, using a stethoscope. Sound analysis was performed and the mean intensity and spectral frequency was obtained.

In this study, 240 single swallowing sounds collected from 20 participants were analyzed by using WaveSurfer (V1.8.8p5; $\mathrm{KTH}$, Stockholm, Sweden). All swallowing sounds consisted of three waves, namely, the first, second, and third swallowing

Table 1. Characteristics of the participants

\begin{tabular}{cc}
\hline Measured item & Mean $\pm \mathrm{SD}$ \\
\hline Age (years) & $29.2 \pm 6.3$ \\
Height $(\mathrm{cm})$ & $169.0 \pm 10.6$ \\
Weight $(\mathrm{kg})$ & $65.2 \pm 16.4$ \\
\hline
\end{tabular}

Values are mean $\pm \mathrm{SD}, \mathrm{n}=20$.

SD: standard deviation.

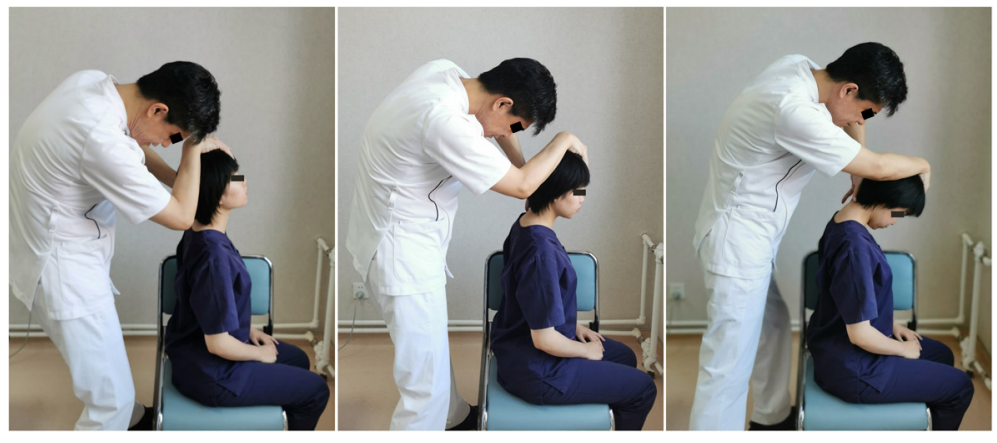

Fig. 1. The neck flexion-lateral flexion-rotation resistance training in NJF neck flexion resistance pattern (NFRP). 
sound wave (SSW), as reported by Honda et $\mathrm{al}^{15}$ ). A representative normal SSW after swallowing $10 \mathrm{~mL}$ of water is shown in Fig. 2. The duration between the beginning of the first SSW and the end of the third SSW was defined as the swallowing sound duration. The mean swallowing sound amplitude and spectral frequency from four trials under three different conditions were analyzed. Afterward, the data were tabulated and analyzed using SPSS (Version 24; IBM, NY, USA). One-way analysis of variance (ANOVA) and Bonferroni multiple comparison test were performed to determine statistical significance.

\section{RESULTS}

This study compared the characteristics of swallowing sounds between NJF-NFRP and Shaker-type exercise through the analysis of swallowing sounds. The results of the one-way ANOVA and Bonferroni multiple comparison test are shown in Table 2 .

The mean swallowing sound amplitude in the NJF-NFRP and Shaker-type conditions was significantly higher than that of the control ( $p<0.01$ and $p<0.05$, respectively). Although the mean amplitude of NJF-NFRP is slightly higher than Shaker-type exercise, there is no significant difference between the two conditions. The mean spectral frequency in the NJF-NFRP and Shaker-type conditions was also significantly higher than that of the control ( $p<0.05$ and $p<0.01$, respectively). The mean spectral frequency amplitude of NJF-NFRP is slightly lower than Shaker-type exercise, but there is no significant difference between the two conditions too. Moreover, no significant differences were observed in the mean swallowing sound duration among the three conditions.

\section{DISCUSSION}

Cervical auscultation is a commonly used method for evaluation in dysphagia. Although there are some shortcomings, this method is simple, non-invasive and can be measured repeatedly, so it has relatively high clinical application value. In addition, in research related to swallowing sound analysis, Shibamoto et al. reported that in a comparison of healthy adults, there is a significant difference in the mean spectral frequency of swallowing sounds between usual swallow and forced swallow ${ }^{16)}$. Babu et al. reported that the amplitude of swallow sound increased significantly with a Shaker-type exercises when compared to usual swallow ${ }^{6}$.

The changes in acoustic characteristics such as the frequency and amplitude of swallowing sounds are correlated with changes in swallowing movements. The increase in amplitude and frequency is probably due to the increased contraction of the muscles related to swallowing movement in the neck flexion exercise such as NFRP and Shaker-type exercises compared to normal swallowing.

Takahashi et al. reported that no significant differences were found for any parameters in both time and frequency domain analyses between swallowing sounds detected bilaterally. Symmetry of the swallowing sounds was verified in the study, and supports the use of "unilateral" site for the detection of swallowing sounds. In addition, the study also suggest that one should evaluate the acoustic characteristics of swallowing sounds from repeated swallows rather than from one swallow ${ }^{14)}$. Therefore, the number of swallowing sound measurements may affect the reliability of this study. Considering that NFRP is a spiral-diagonal resistance training, and the left and right is performed one after the other. Therefore, in the present study in order to ensure the repeatability and reliability of the trial, the swallowing sounds were recorded twice on each side of the neck, and the average value of these 4 swallowing sounds was used for statistical analysis.

The Shaker exercise is known to effectively strengthen the suprahyoid and infrahyoid muscles ${ }^{1)}$, thereby improving superior and anterior laryngeal movement and widening the UES opening. These effects explain why strengthening the muscles is particularly important in dysphagia rehabilitation.

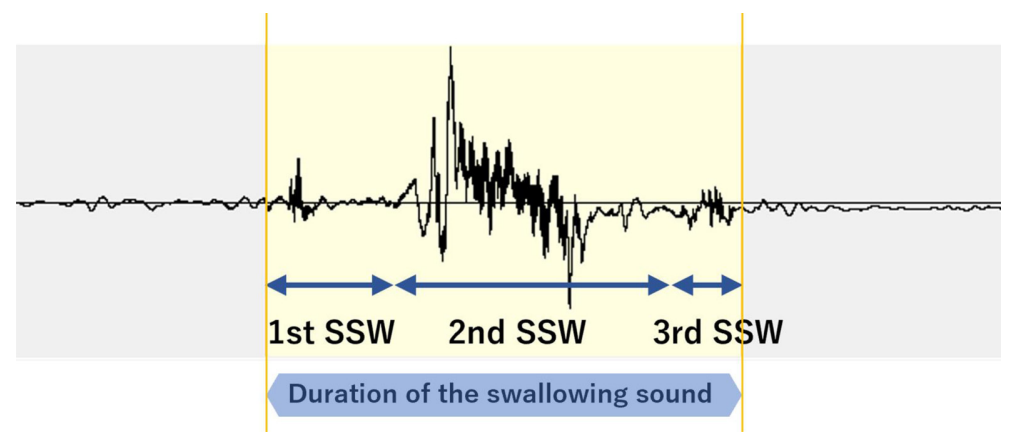

Fig. 2. Normal swallowing sound waveform after swallowing $10 \mathrm{~mL}$ of water.
Table 2. Swallowing sound characteristics

\begin{tabular}{ll}
\hline The mean amplitude $(\mathrm{dB})$ & \\
NJF & $44.0 \pm 2.3$ \\
Shaker & $43.6 \pm 2.7$ \\
control & $42.2 \pm 1.8$ \\
The mean frequency $(\mathrm{Hz})$ & \\
NJF & $241.5 \pm 37.8$ \\
Shaker & $253.3 \pm 29.3$ \\
control & $217.6 \pm 22.1$ \\
The mean duration (ms) & \\
NJF & $709.6 \pm 44.2$ \\
Shaker & $705.8 \pm 36.7$ \\
control & $719.6 \pm 29.4$ \\
\hline \multicolumn{2}{l}{ Values are mean $\pm \mathrm{SD}, * \mathrm{p}<0.05, * * \mathrm{p}<0.01}$. \\
NJF: neuromuscular joint facilitation; Shaker: \\
Shaker-type exercise; SD: standard deviation.
\end{tabular}


However, patients with insufficient motor function, such as hemiplegia, may have difficulty in completing the Shaker exercise by themselves. The intensity of the exercise has been known to be physically challenging for elderly individuals ${ }^{7}$.

We tried to better adapt to the patients' physical motor function, in collaboration with a physical therapist, to accommodate those with difficulties in performing the exercise for various reasons, including aging. We hypothesized that the use of manual therapy techniques for neck flexion resistance exercise training (i.e., NJF) can effectively stimulate the suprahyoid and infrahyoid muscles in such individuals.

The effectiveness of head rotation as a compensatory technique for safe swallowing has also been reported ${ }^{17)}$. In addition, swallowing exercises, such as neck flexion, rotation, and lateral flexion, can improve dysphagia symptoms and prevent complications $^{18)}$. Pre-prandial swallowing exercises, which include a series of neck movements to effectively strengthen related muscles and slow down degenerative changes, can help reduce the risk of dysphagia ${ }^{18)}$. Moreover, a study conducted in Japan reported that kyphotic patients also have difficulties in maintaining a supine position ${ }^{8)}$. Thus, these factors should also be considered.

NJF is a relatively new therapeutic technique. It has shown relatively good therapeutic effects in recent clinical applications, especially in the improvement of physical motor function, balance, and pain ${ }^{19-21)}$. It has a relatively wide range of applications in terms of target populations. Training is based on spiral-diagonal movement patterns. In NJF-NFRP training, elements of neck flexion, lateral flexion, and rotation with resistance are combined. Therefore, we hypothesized that the training method is more conducive in stimulating suprahyoid, infrahyoid, and/or pharyngeal muscle contraction, subsequently improving swallowing functions.

To our knowledge, there have been no studies on the effect of NJF treatment on swallowing function. In cooperation with a team of physical therapists, the authors examined whether NJF-NFRP can effectively help people with swallowing problems. We expected that the technique would benefit individuals with insufficient UES opening due to suprahyoid muscle weakness, and insufficient laryngeal movement, as well as those who have difficulty completing the Shaker exercise.

The effect of PNF technique on swallowing functions has also been reported. Kim et al. reported that PNF-based short neck flexion exercises as treatment for swallowing disorders in stroke patients were as effective as the Shaker exercise ${ }^{22)}$. Sayaca et al. reported that the PNF isotonic neck flexion treatment increased the peak contraction amplitude of suprahyoid muscles more than the Shaker exercise ${ }^{23)}$. Compared with the PNF neck pattern, the NJF-NFRP exerts resistance not only on the forehead, but also on the proximal joints of the cervical spine simultaneously. Currently, the differences of the two techniques in terms of axis and torque influence on the force applied to the suprahyoid and infrahyoid muscles remain unclear. Further studies on the differences between the neck patterns of PNF-based and the NJF-based treatment methods are needed.

Compared to the Shaker exercise, the NJF-NFRP is a combination of neck flexion, lateral flexion, and rotation with resistance, with similar characteristics on acoustic analysis. Therefore, our results imply that the NJF-NFRP would be a promising rehabilitation technique in managing issues related to the pharyngeal and suprahyoid muscle contraction.

Our study has some limitations. First, the swallowing sound of healthy individuals was evaluated. Further studies including individuals with swallowing disorders are recommended. Second, although the limb posture and intervention intensity have been revised to minimize the errors caused by the difference in limb posture and intensity between the two methods, there is indeed a difference in the posture of the two methods during the measurement. Therefore, this measurement error still exists in the present study. In order to improve this shortcoming, it is necessary to compare before and after the intervention in further studies. Third, only the immediate effects of the NJF-NFRP had been examined. Long-term effects of NJF-NFRP intervention on swallowing functions need to be investigated in the future. Fourth, the swallow sound contained audible cues; therefore, it was possible to find some characteristics of swallowing movements through acoustic analysis. Reliability of cervical auscultation is insufficient when used as a stand-alone diagnostic tool ${ }^{24,25)}$; thus, it is necessary that further studies combine cervical auscultation with other assessment methods, such as electromyography and video fluoroscopy, for verification of the sound characteristics.

We analyzed the effect of NJF-NFRP treatment on the swallowing sounds of healthy adults using cervical auscultation. Though this is a preliminary study, the results indicated that NJF-NFRP training exhibited remarkable influences on the swallowing sounds in healthy adults. The acoustic characteristics obtained during NJF-NFRP treatment were similar to those of the Shaker-type exercises, indicating that NJF-NFRP may be as helpful as Shaker-type exercises in enhancing pharyngeal and suprahyoid muscle contraction.

Funding

The authors received no specific funding for this work.

Conflict of interest

The authors declare no conflicts of interest. 


\section{REFERENCES}

1) Woo HS, Won SY, Chang KY: Comparison of muscle activity between two adult groups according to the number of Shaker exercise. J Oral Rehabil, 2014, 41: 409-415. [Medline] [CrossRef]

2) Shaker R, Easterling C, Kern M, et al.: Rehabilitation of swallowing by exercise in tube-fed patients with pharyngeal dysphagia secondary to abnormal UES opening. Gastroenterology, 2002, 122: 1314-1321. [Medline] [CrossRef]

3) Shaker R, Kern M, Bardan E, et al.: Augmentation of deglutitive upper esophageal sphincter opening in the elderly by exercise. Am J Physiol, 1997, 272: G1518-G1522. [Medline]

4) Logemann JA, Rademaker A, Pauloski BR, et al.: A randomized study comparing the Shaker exercise with traditional therapy: a preliminary study. Dysphagia, 2009, 24: 403-411. [Medline] [CrossRef]

5) Choi JB, Shim SH, Yang JE, et al.: Effects of Shaker exercise in stroke survivors with oropharyngeal dysphagia. NeuroRehabilitation, 2017 , 41: 753-757. [Medline] [CrossRef]

6) Babu S, Balasubramaniam RK, Varghese A: Effect of modified Shaker exercise on the amplitude and duration of swallowing sounds: evidence from cervical auscultation. Rehabil Res Pract, 2017, 2017: 6526214. [Medline]

7) Easterling C, Grande B, Kern M, et al.: Attaining and maintaining isometric and isokinetic goals of the Shaker exercise. Dysphagia, 2005, 20: 133-138. [Medline] [CrossRef]

8) Oshima F, Fujiu-Kurachi M, Fujishima I: Rehabilitation for dysphagia. Larynx, 2020, 32: 20-28. [CrossRef]

9) Yoon WL, Khoo JK, Rickard Liow SJ: Chin tuck against resistance (CTAR): new method for enhancing suprahyoid muscle activity using a Shaker-type exercise. Dysphagia, 2014, 29: 243-248. [Medline] [CrossRef]

10) Fujiki RB, Oliver AJ, Malandraki JB, et al.: The recline and head lift exercises: a randomized clinical trial comparing biomechanical swallowing outcomes and perceived effort in healthy older adults. J Speech Lang Hear Res, 2019, 62: 631-643. [Medline] [CrossRef]

11) Huo M: Neuromuscular joint facilitation. Tokyo: Ipec press, 2010, pp 127-138.

12) Pan Q, Maeda N, Manda Y, et al.: Validation of the optimal site in the neck region for detecting swallowing sounds. J Oral Rehabil, 2016, 43: 840-846. [Medline] [CrossRef]

13) Takahashi K, Groher ME, Michi K: Methodology for detecting swallowing sounds. Dysphagia, 1994, 9: 54-62. [Medline] [CrossRef]

14) Takahashi K, Groher ME, Michi K: Symmetry and reproducibility of swallowing sounds. Dysphagia, 1994, 9: 168-173. [Medline] [CrossRef]

15) Honda T, Baba T, Fujimoto K, et al.: Characterization of swallowing sound: preliminary investigation of normal subjects. PLoS One, 2016, 11: e0168187. [Medline] [CrossRef]

16) Shibamoto I: The volitional control of swallowing sounds in elders. Asia Pacific Conference of Speech Language and Hearing, 2015,2015 : PS-2-015.

17) Logemann JA, Kahrilas PJ, Kobara M, et al.: The benefit of head rotation on pharyngoesophageal dysphagia. Arch Phys Med Rehabil, 1989, 70: 767-771. [Medline]

18) Nagao N, Tanaka N, Fujishima I, et al.: Improvement in dysphagia symptoms by continuous practice of the Fujishima-style swallowing exercise set. Deglutition, 2018, 7: 262-272.

19) Huo M, Maruyama H, Kaneko T, et al.: The immediate effect of lumbar spine patterns of neuromuscular joint facilitation in young amateur baseball players. J Phys Ther Sci, 2013, 25: 1523-1524. [Medline] [CrossRef]

20) Onoda K, Huo M, Maruyama H: The immediate effect of neuromuscular joint facilitation (NJF) treatment on the standing balance in younger persons. J Phys Ther Sci, 2015, 27: 1481-1483. [Medline] [CrossRef]

21) Huang Q, Li D, Zhang Y, et al.: The intervention effects of different treatments for chronic low back pain as assessed by the thickness of the musculus transversus abdominis. J Phys Ther Sci, 2014, 26: 1383-1385. [Medline] [CrossRef]

22) Don Kim K, Lee HJ, Lee MH, et al.: Effects of neck exercises on swallowing function of patients with stroke. J Phys Ther Sci, 2015, 27: 1005-1008. [Medline] [CrossRef]

23) Sayaca C, Serel-Arslan S, Sayaca N, et al.: Is the proprioceptive neuromuscular facilitation technique superior to Shaker exercises in swallowing rehabilitation? Eur Arch Otorhinolaryngol, 2020, 277: 497-504. [Medline] [CrossRef]

24) Lagarde ML, Kamalski DM, van den Engel-Hoek L: The reliability and validity of cervical auscultation in the diagnosis of dysphagia: a systematic review. Clin Rehabil, 2016, 30: 199-207. [Medline] [CrossRef]

25) Leslie P, Drinnan MJ, Finn P, et al.: Reliability and validity of cervical auscultation: a controlled comparison using videofluoroscopy. Dysphagia, 2004, 19: 231-240. [Medline] 